\title{
Performance Evaluation of Image Enhancement Techniques
}

\author{
Shruti Puniani ${ }^{1}$ and Sankalap Arora ${ }^{2}$ \\ ${ }^{1}$ Research Scholar, Dept. of Computer Science, \\ DAV University, Jalandhar, Punjab, India \\ ${ }^{2}$ Assistant Professor, Dept. of Computer Science, \\ DAV University, Jalandhar, Punjab, India \\ ${ }^{1}$ Puniani.shruti@gmail.com, ${ }^{2}$ Sankalap.arora@gmail.com
}

\begin{abstract}
Color Image enhancement is a process in which the perceptual information of an image is improved to obtain more information and details contained in the image. It improves the subjective quality of an image by working with original data. This paper focuses on evaluating the performance of various image enhancement techniques. These techniques are either based on histogram modification or are based on fuzzy logic. The techniques are compared using two quantitative measures namely; Contrast Improvement index (CII) and Tenengrad measure. The results have shown that Lab and edge preservation based fuzzy image enhancement (LEFM) yields the best results.
\end{abstract}

Keywords: Color image, fuzzy logic, Lab color space, Histogram equalization, edge preserving smoothing

\section{Introduction}

Image enhancement techniques play an important role to improve the visual appearance of a digital image so that extracting image details becomes easy [1]. These techniques have varied applications in medical image processing like detection of cancers, tumors etc., seismic exploration, video processing, camera and surveillance. Various image enhancement techniques have been developed so far in literature, such as gray-level transformation based techniques (e.g. logarithmic transformation, power-law transformation, piecewise-linear transformation, etc.) and histogram based techniques (e.g. Histogram equalization, histogram specification, etc.) [2]. The ordinary image enhancement methods fail to provide satisfying results when applied on low-contrast images. This is because in low contrast images, the component of histogram is higher at one end and lower at another end of the histogram. So, it is difficult to balance its contrast by using gray-level transformation methods [3]. Also, these techniques cause washed out appearance by affecting its background. Histogram specification suffers from a major flaw that the histogram of the final image has to be established manually. Thus, it is not an automatic method [4]. Various techniques have evolved from histogram equalization, such as Bi-Histogram Equalization (BHE), Adaptive Histogram Equalization (AHE), Dualistic Sub-image Histogram Specification (DSIHE) etc. AHE divides the original image into a cluster of sub-images and then apply histogram equalization on each sub-image individually [5]. After this, the sub-images are merged together using bilinear interpolation.

Image enhancement using fuzzy-logic has gained a lot of attention in recent years. A digital image with gray tone suffers from ambiguity and vagueness [6]. This vagueness is defined using linguistic variables, such as 'good contrast', 'light red' etc. An image can be thought as an array of fuzzy singletons each having a membership function specifying the varying degree of brightness levels [7]. Fuzzy image processing consists of three steps: (1) Image fuzzification in which the image in spatial domain is converted 
into fuzzy membership domain (2) Modification of membership values; and (3) Image de-fuzzification in which the image in fuzzy domain is re-converted into spatial domain for the viewer. The fuzzy membership values are modified using some fuzzy rules, fuzzy clustering, etc.

This paper is organized as Section 2 discusses the research papers studied while carrying out a survey. Section 3 deals with theoretical analysis of various techniques. Section 4 explains evaluation metrics and section 5 deals with experimental analysis and results. In the end, the paper is concluded in Section 6.

\section{Related Work}

Chaira T. has suggested a Type II fuzzy set theory for carrying out image enhancement. Type II fuzzy set is required so as to get better information on uncertainty on membership function. Hamacher T co norm is used as aggregation operator to create a new membership function using upper and lower membership function of Type II fuzzy set. It is basically used for enhancing medical images since they contain uncertainties. This method is compared with fuzzy, intuitionistic fuzzy, and existing Type II fuzzy methods. The experimental analysis shows that Type II fuzzy set given in this paper yields better results than other methods. Using this method, abnormal lesions can be detected and extracted from the enhanced images [8].

De Araujo et al. have developed a nature inspired image enhancement technique which is based on artificial life model. According to this model, a herbivore organism selects its food when it is in a suitable environment. It selects the food in iterations and travels from one point to another in an image. The simulations of its activities and and outcomes on the surroundings have been analyzed in this. The technique is tested on low contrast images having various levels of noise in them. The results prove that artificial life model has improved the contrast of images [9].

Zhou, Zhigang et al. have proposed a new adaptive method to improve the contrast of low bright and low contrast images. The algorithm can be defined in three steps: (1) global brightness adaptive adjustment (2) locally adaptive contrast enhancement and (3) color restoration. Studies reveal that the illumination and local contrast of low illumination color image may be successfully enhanced. The important points, particularly in dark region of enhanced tend to be more distinguished and the enhanced image is more brilliant than several other techniques for low illumination color image enhancement [10].

A. K. Bhandari et al. have examined a new approach based on Cuckoo Search (CS) and DWT-SVD to improve the quality of low contrast satellite images. Firstly, the original image is decomposed into four frequency sub-bands using DWT and CS method is used to optimize each sub-band, then a singular matrix of low-low sub-band is obtained. In the end, the enhanced image is reconstructed using inverse discrete wavelet transform (IDWT). The singular matrix is responsible for changing the intensity information of the image [11].

Raju, G., and Madhu S. Nair have discussed a fast and reliable method for enhancing low contrast and low bright color images using fuzzy-logic and histogram. They have mainly focused on converting skewed histogram into a uniform histogram. The input RGB image is converted into HSV so as to stretch the V component preserving the chromatic information. The enhancement of $\mathrm{V}$ is done using two parameters i.e. contrast intensification parameter and average intensity value of the image. The technique is comparatively fast when compared to existing techniques [12].

$\mathrm{Li}$, Jia et al. have image enhancement methods on the basis of various factors such as contrast enhancement, linear transformation, piecewise linear transformation and retinex algorithms. The implementation of the Retinex method has been analyzed and it has 
shown that the technique can realize color constancy, color enhancement and overall dynamic range compression in certain situations [13].

\section{Theoretical Analysis}

\subsection{Histogram Equalization (HE)}

Histogram Equalization is a spatial domain technique which is very popular because it is simple and easy to implement [14]. It helps to modify intensity of an image to enhance its contrast. It is a transformation which extends the contrast of an image by redistributing the gray level uniformly. It can be represented with the help of an equation as follows:

$$
\mathrm{P}\left(\mathrm{r}_{\mathrm{k}}\right)=\frac{\mathrm{n}_{\mathrm{k}}}{\mathrm{n}}
$$

where $\mathrm{n}_{\mathrm{k}}=0,1,2 \ldots \ldots, \mathrm{L}-1$ and $\mathrm{r}_{\mathrm{k}}$ is the kth gray level and $n$ is the number of pixels in image having gray level $\dot{\Gamma}_{k}$. Though the technique is simple yet its conventional nature leads to unnatural look of over enhancement. The major drawback of Histogram Equalization is that it gives washed out appearance to an image [15]. Also, it only focuses on the global contrast enhancement of the image leading to loss of local details which can also lead to over enhancement of an image.

\subsection{Adaptive Histogram Equalization (AHE)}

This technique works by transforming every pixel in an image using a transformation function which is produced from a neighborhood area. It improves the local contrast of an image [16]. The neighborhood area of a pixel consists of a scale in which contrast of a pixel in smaller scale is improved while contrast at larger scale is reduced. This method is basically used to enhance both natural and medical images. But is may over amplify noise in homogenous regions. Also, due to poor and non uniform lighting conditions of objects and non-linearity of imaging system, vagueness is introduced in the final image. This vagueness appears in the form of imprecise boundaries and color values during image digitization.

\subsection{Image Enhancement based on Fuzzy -logic}

Fuzzy-logic has been successfully employed in various areas of Image processing. Fuzzy image processing solves the problem of vagueness which is produced in methods discussed above [17]. It is solved using fuzzy sets and Linguistic variables such as 'good contrast' or 'sharp boundaries', 'light red', 'dark green', etc [18]. According to recent studies, fuzzy-logic based techniques have proved to be better than traditional methods of image enhancement. Fuzzy image processing consists of three main steps: (1) Image fuzzification in which spatial domain is converted into fuzzy membership domain; (2) modification of membership values using Fuzzy rules; and (3) image de-fuzzification in which fuzzy membership domain is re-converted into spatial domain. In the fuzzy method, firstly, RGB image is converted into HSV in which V component is stretched and $\mathrm{H}$ and $\mathrm{S}$ are not changed. The membership function is modified depending upon two parameters $\mathrm{X}$ and $\mathrm{Y}$ where $\mathrm{X}$ is average intensity value of the image and $\mathrm{Y}$ is contrast intensification parameter. The value of $X$ can be calculated using eq. (2)

$$
X=\frac{\sum_{x} x G(x)}{\sum_{x} G(x)}
$$


Where $G(x)$ is histogram which means number of pixels in the image with intensity value ' $x$ '.

The parameter $\mathrm{X}$ divides the histogram into two classes: $\mathrm{X}_{1}$ and $\mathrm{X}_{2}$. The pixels in $\mathrm{X}_{1}$ lie in the range [0, $\mathrm{X}-1]$ and pixels in $\mathrm{X}_{2}$ lie in the range [X, 255].The $\mathrm{V}$ component is stretched on the basis of two membership values $\alpha_{1}$ and $\alpha_{2}$. The fuzzy membership values are modified on the basis of fuzzy rules explained below:

1. If the difference between $x$ and $X$ is large, then the intensity of stretching should be small. To implement this rule and to calculate value of $\alpha_{1}$ eq. (3) is used.

$$
\alpha_{1}=\frac{1-((X-x)}{X}
$$

This rule states that pixel values closer to $X$ will stretched more while values farther from $X$ will be stretched lesser. The fuzzy membership value $\alpha_{2}$ for class $X_{2}$ is based on following fuzzy rule:

2. If the difference between $\mathrm{x}$ and $\mathrm{D}$ is large, then the intensity of stretching should be large. It states that pixel values closer to $\mathrm{D}$ will be stretched lesser while values farther from $\mathrm{D}$ will be stretched more. To implement this fuzzy rule and to calculate $\alpha_{2}$ eq. (4) is used. ( $\mathrm{D}$ is extreme value e.g. $\mathrm{D}=255$ for 8-bit images)

$$
\alpha_{2}=\frac{D-x}{D-X}
$$

The major drawback of this method is that it is only suitable for low contrast and low bright images. Also, the value of $\mathrm{K}$ is fixed in this. The fuzzy based method doesn't focus on edge preservation which gets affected during image processing.

\subsection{Lab and Edge Preservation based Fuzzy Image Enhancement (LEFM)}

This method has been developed by integrating the above explained fuzzy based method with edge preserving smoothing. It uses $\mathrm{L}^{*} \mathrm{a} * \mathrm{~b} *$ color space which is device independent. The RGB image is converted into Lab color space because RGB model contains only color components and no light channel. Thus, to preserve the color information, it is converted into Lab model in which L is light channel and a and $\mathrm{b}$ are color components. Further, edge preserving smoothing [19] is applied on fuzzy image enhancement so that the edges are not affected and remain preserved. It can be done in following way:

Firstly, a small square sliding window of length $\mathrm{Z}$ is taken. It has been proved that a small square window yields best possible results in terms of preserving edges and fine details. The value of $Z$ can be evaluated using eq. (5)

$$
\mathrm{Z}=\left\{\begin{array}{l}
3, \mathrm{n}<0.5 \\
5,0.5 \leq \mathrm{n} \leq 0.6
\end{array}\right.
$$

where $\mathrm{n}$ is noise density which can be calculated using eq. (6)

$$
\mathrm{n}=\frac{\mathrm{L}}{\mathrm{RC}}
$$

where $\mathrm{L}$ is total number of zeros and 255 in the image and product $\mathrm{RC}$ is number of pixels in image. Then, the most optimum value of a threshold $\beta$ is evaluated by using eq. (7) where $\beta$ is a threshold which depends on noise density and characteristics of an image and $\beta \leq \mathrm{Z}^{2}$.

$$
\beta= \begin{cases}\left\lfloor Z^{2}(n+0.50)\right\rfloor & Z=3 \\ \left\lfloor Z^{2}(n+0.15)\right\rfloor & Z=5\end{cases}
$$

where $[$.$] is the floor operation.$ 


\section{Evaluation Metrics}

To compare different image enhancement techniques, quantitative performance measures have been used.

\subsection{Contrast Improvement Index (CII)}

This is the most important benchmark to compare the performance of various image enhancement techniques. It can be measured as a ratio of local contrast of final and input images. It can be represented using eq. (8).

$$
\mathrm{CII}=\frac{\mathrm{A}_{\text {proposed }}}{\mathrm{A}_{\text {original }}}
$$

where $A$ is the average value of the local contrast measured with $3 \times 3$ window.

$A_{\text {proposed }}$ and $A_{\text {original }}$ are the average values of the local contrast in the output and original images, respectively. If the value of CII increases, then it shows improvement in contrast of an image.

\subsection{Tenengrad Measure}

This measure is based on gradient measure maximization. If the value of Tenengrad is larger, then the image quality is considered to be higher. Also, the Tenengrad measure of different images and different techniques is different since the intensity of different images is different. The Tenengrad is based on gradient magnitude maximization. It is recognized as one of the most precise and powerful image quality measures. The Tenengrad value of an image $\mathrm{J}$ is calculated from the gradient $\Delta \mathrm{J}(\mathrm{x}, \mathrm{y})$ where the partial derivatives are calculated using a sobel filter having convolution kernels $j_{\mathrm{x}}$ and $\mathrm{j}_{\mathrm{y}}$. The gradient magnitude is calculated using eq. (9)

$$
T(x, y)=\sqrt{\left(j_{x} \otimes J(x, y)\right)^{2}+\left(j_{y} \otimes J(x, y)\right)^{2}}
$$

and the Tenengrad is measured using eq. (10)

$$
\mathrm{TGD}=\sum_{\mathrm{x}} \sum_{\mathrm{y}} \mathrm{T}(\mathrm{x}, \mathrm{y})^{2}
$$

The quality of an image is considered to be high if its Tenengrad value is high. It basically signifies that structural information of the image is preserved.

\section{Experiments}

For the experiments, the Matrix Laboratory (MATLAB) image processing toolbox is used for designing and implementation of all algorithms.

\subsection{Experimental Setup}

To implement the algorithm, four different color images namely; Fields, Beach, grapes and stones are taken. Figure 1 shows the input images taken and Figure 2 shows the visual results obtained after applying image enhancement techniques.

Various image enhancement techniques are applied on these images on the basis of performance measures discussed earlier. The techniques are:

1. Histogram Equalization (HE)

2. Adaptive histogram equalization (AHE)

3. Fuzzy based method

4. Lab and Edge preservation based Fuzzy image enhancement (LEFM) 


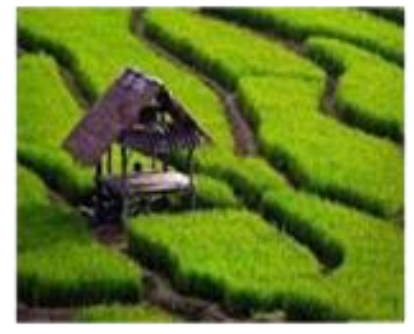

(a) Fields

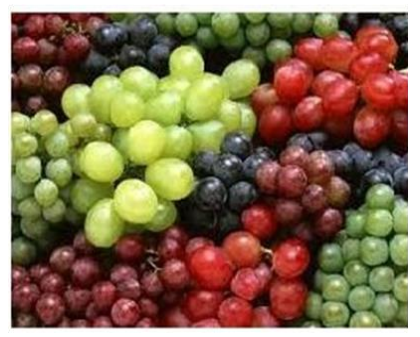

(c) Grapes

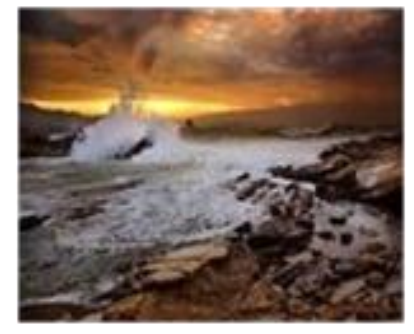

(b) Beach

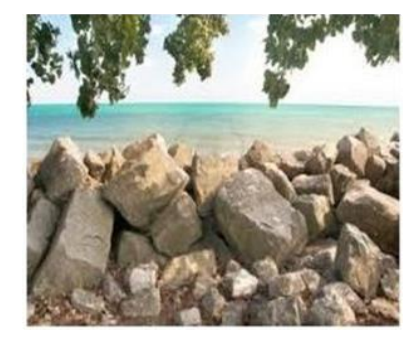

(d) Stones

Figure 1. Original Images

\subsection{Results and Discussion}

After applying various image enhancement algorithms on color images, following results have been obtained. The comparison of these algorithms is done on the basis of evaluation metrics.

5.2.1 Contrast Improvement Index (CII): Figure 3 shows graphical representation of CII which is obtained after applying various techniques on color images. From the figure, it can be inferred that histogram equalization (HE) and adaptive histogram equalization (AHE) yield very less values of CII. It means that they hardly improve the contrast of an image. This is because of the fact that HE only focuses on global contrast of image leading to loss of local details. Also, in AHE due to poor and non-uniform lightning of imaging system, vagueness is introduced in image which appears in the form of imprecise boundaries and color values during digitization. The fuzzy method gives better CII values as compared to HE and AHE because it solves the problem of imprecise boundaries and vagueness using fuzzy sets and linguistic variables. But it cannot perform well enough when compared with LEFM because it suffers from edge degradation problem which may arise while applying image enhancement algorithm. 


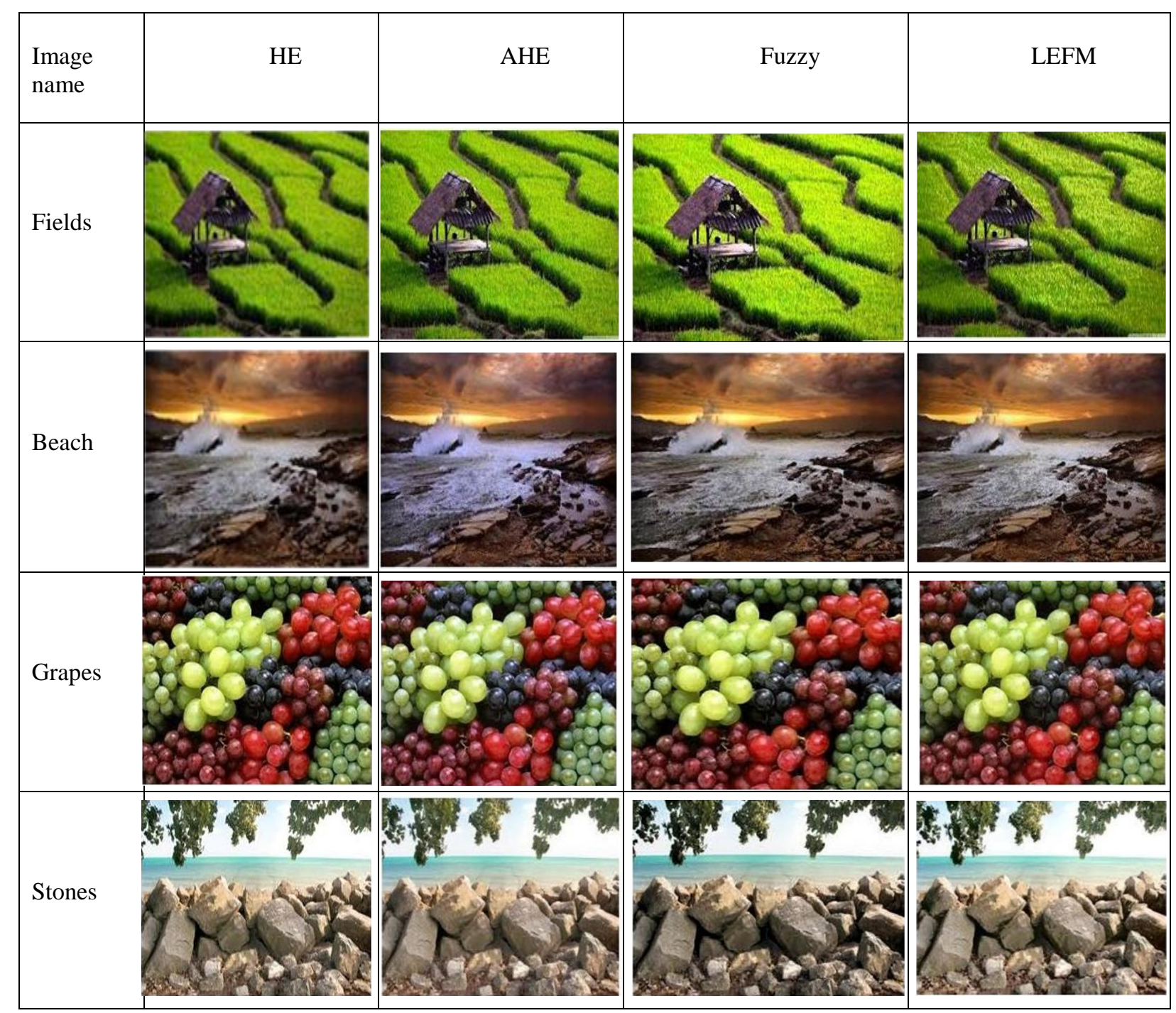

Figure 2. Visual Results Obtained after Applying Different Enhancement Techniques

On the contrary, LEFM solves this problem using edge preserving smoothing algorithm which is integrated with Fuzzy method. So, it enhances an image while preserving edges and local details. Also, it uses Lab color space which is device independent due to which the contrast or brightness of image don't get affected while using same image on different machines. Thus, it yields highest CII values in all images proving the fact that contrast has been improved in case of LEFM. 


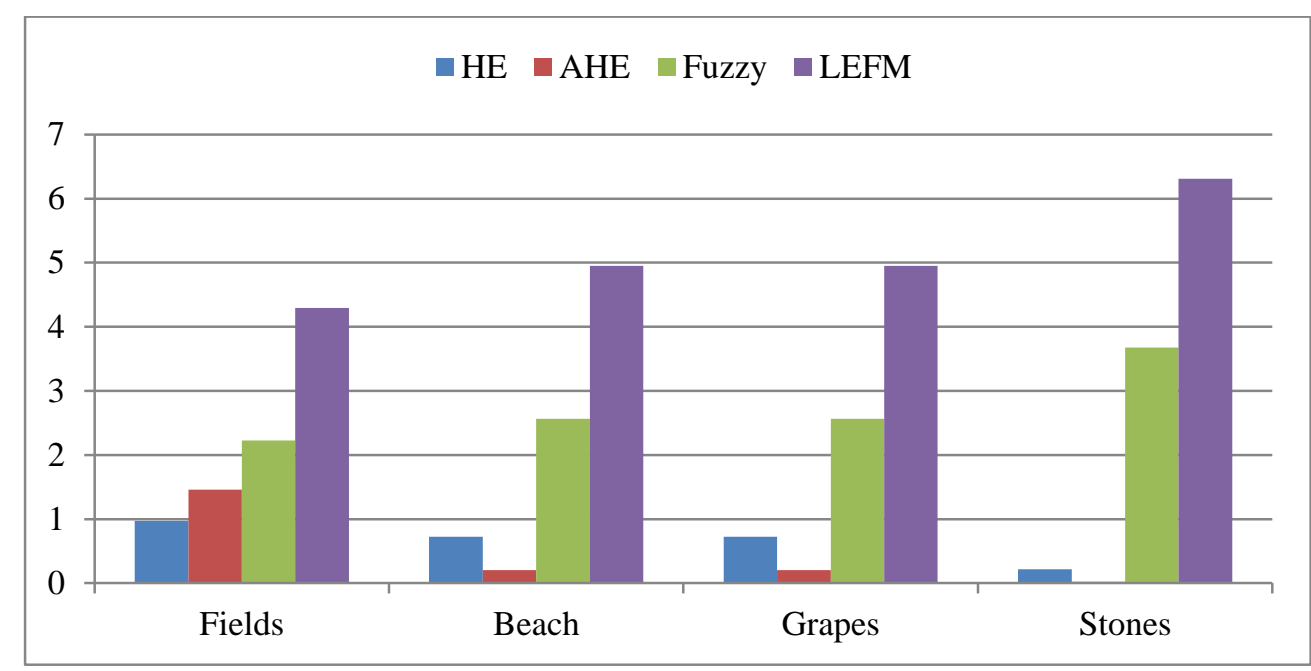

Figure 3. CII Evaluation of HE, AHE, Fuzzy and LEFM

Table 1 shows the numerical values of CII obtained after applying various techniques on the input images. LEFM produces highest values of CII in all images (represented in bold) proving the fact that it is a better technique than state-of-the-art-techniques.

Table 1. Cll Analysis

\begin{tabular}{|c|c|c|c|c|}
\hline $\begin{array}{c}\text { Image } \\
\text { name }\end{array}$ & HE & AHE & Fuzzy & LEFM \\
\hline Fields & 0.7881 & 2.3883 & 3.3753 & $\mathbf{4 . 8 5 9 6}$ \\
\hline Beach & 0.9690 & 1.4612 & 2.2261 & $\mathbf{4 . 2 9 3 0}$ \\
\hline Grapes & 0.7227 & 0.2025 & 2.5661 & $\mathbf{4 . 9 4 9 1}$ \\
\hline Stones & 0.2174 & 0.0247 & 3.6758 & $\mathbf{6 . 3 0 8 6}$ \\
\hline
\end{tabular}

5.2.2 Tenengrad Measure: It is the most robust and functionally accurate image quality measure. It is used to analyze whether structural information of an image is improved or not. Structural information is an idea that pixels have strong interdependencies especially when they are spatially close. These dependencies carry important information about structure of objects in visual scene. Figure 4 shows the chart showing values of Tenengrad measure after applying different techniques on color images. From the chart, it can be deduced that HE produces the least values of Tenengrad because it doesn't focus on local contrast of images. So, some regions remain unaffected because local regions don't get modified. Thus, structural information is lost. AHE usually leads to loss of edges of objects and over-enhancement of noise in images due to which pixels get corrupted due to noise. Thus, it also produces lesser Tenengrad values as compared to fuzzy and LEFM. The fuzzy method fixes this problem using fuzzy sets and linguistic variables. Also, it mainly focuses on modifying local contrast of images. Thus, structural information is preserved. But it doesn't yield better values than LEFM because it uses HSV color space which is device dependent and also doesn't focus on preserving edges leading to loss of structural details. On the other hand, LEFM outperforms all these techniques because it deals with all these shortcomings. It uses edge preserving smoothing algorithm which uses a sliding window to filter out noise. Also, it uses Lab color model which is device independent. Thus, pixels have strong inter-dependencies which preserve important information of objects in visual scene making image processing operations easy. 


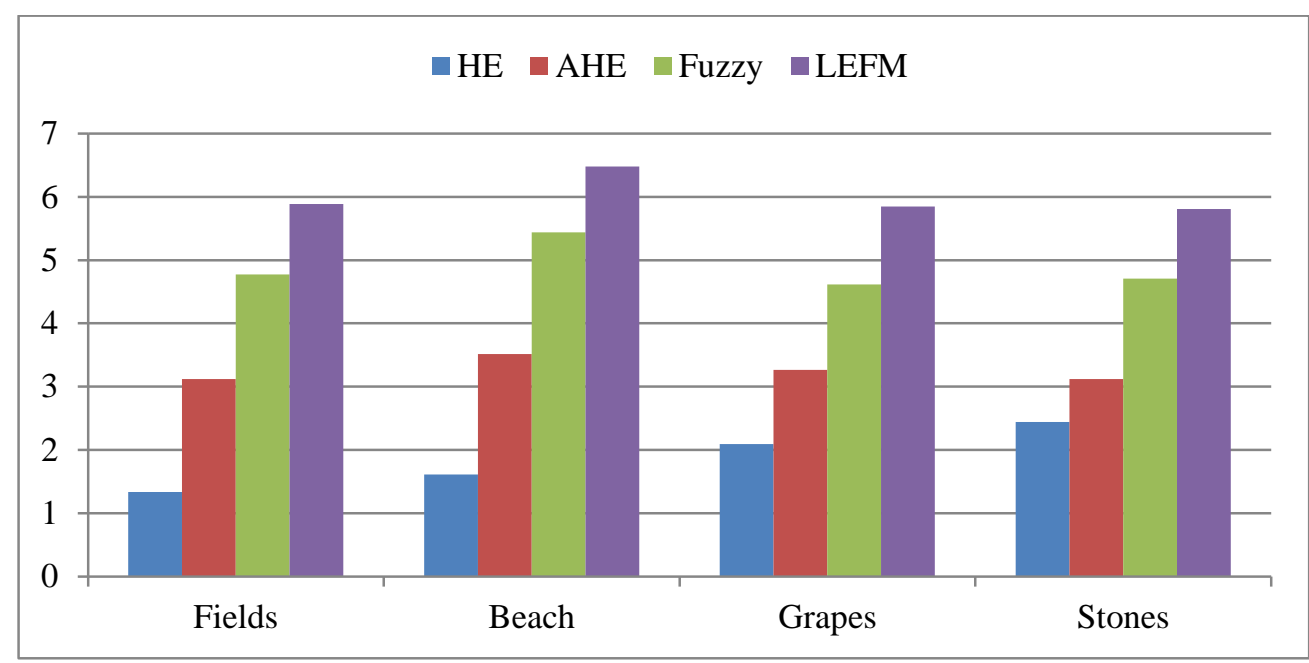

Figure 4. Tenengrad Analysis of HE, AHE, Fuzzy and LEFM

Table 2 shows the quantitative values obtained after applying various techniques on color images. LEFM outperforms the existing techniques indicating that it preserves the structural information in a better way.

Table 2. Tenengrad Measure

\begin{tabular}{|c|c|c|c|c|}
\hline $\begin{array}{c}\text { Image } \\
\text { name }\end{array}$ & HE & AHE & Fuzzy & LEFM \\
\hline Fields & 1.3341 & 3.1187 & 4.7722 & $\mathbf{5 . 8 8 5}$ \\
\hline Beach & 1.6093 & 3.5179 & 5.4371 & $\mathbf{6 . 4 7 9 9}$ \\
\hline Grapes & 2.0951 & 3.2662 & 4.6140 & $\mathbf{5 . 8 4 7 1}$ \\
\hline Stones & 2.4381 & 3.1201 & 4.7055 & $\mathbf{5 . 8 0 5 9}$ \\
\hline
\end{tabular}

\section{Conclusion}

In this paper, performance of different image enhancement algorithms has been tested on color images. To compare these algorithms, Contrast Improvement Index (CII) and Tenengrad have been used as performance metrics. It can be concluded that $\mathrm{L}^{*} \mathrm{a} \mathrm{b}^{*}$ color space and edge preservation based fuzzy image enhancement (LEFM) has produced the best result out of HE, AHE and fuzzy based image enhancement. This is because of the fact that this method uses $\mathrm{L}^{*} \mathrm{a}^{*} \mathrm{~b}^{*}$ color space which is device independent color space. Due to this, the contrast and brightness of an image is not dependent on which it is produced. Thus, the quality of an image is not dependent on the device on which it is taken and the device on which it is modified. Also, this method has been integrated with an edge preserving smoothing algorithm which helps in enhancement of image while preserving its edges. This further helps in other image processing operations such as segmentation, edge detection etc. Thus, detecting cancers and tumours becomes easy. Also, it helps to enhance the medical and X-ray images. 


\section{References}

[1] Negi, Shailendra Singh, and Yatendra Singh Bhandari. "A hybrid approach to Image Enhancement using Contrast Stretching on Image Sharpening and the analysis of various cases arising using histogram." In Recent Advances and Innovations in Engineering (ICRAIE), 2014, pp. 1-6. IEEE, 2014.

[2] Chen, ZhiYu, "Gray-level grouping (GLG): an automatic method for optimized image contrast Enhancement-part I: the basic method." Image Processing, IEEE Transactions pp.2290-2302, 2006.

[3] Bhattacharya, Saumik, Sumana Gupta, and Venkatesh K. Subramanian. "Localized image enhancement." Communications (NCC), 2014 Twentieth National Conference on. IEEE, 2014.

[4] Senthilkumaran, N., and J. Thimmiaraja. "Histogram equalization for image enhancement using MRI brain images." Computing and Communication Technologies (WCCCT), 2014 World Congress on. IEEE, 2014.

[5] Kathiravan, R., R. Shanmugasundaram, and N. Santhiyakumari. "Satellite image resolution enhancement using contrast limited adaptive histogram equalization." Digital Image Processing,pp.161165,2014

[6] Hanmandlu, Madasu, and Devendra Jha. "An optimal fuzzy system for color image enhancement." Image Processing, IEEE Transactions pp. 2956-2966, 2006.

[7] Pal, Sankar K., and R. King. "Image enhancement using smoothing with fuzzy sets." IEEE TRANS. SYS., MAN, AND CYBER., pp. 494-500,1981.

[8] Chaira, Tamalika. "An improved medical image enhancement scheme using Type II fuzzy set." Applied Soft Computing .pp.293-308,2014.

[9] de Araujo, Alex F., Christos E. Constantinou, and João Manuel RS Tavares. "New artificial life model for image enhancement." Expert Systems with Applications,pp. 5892-5906,2014.

[10] Zhou, Zhigang, Nong Sang, and Xinrong Hu. "Global brightness and local contrast adaptive enhancement for low illumination color image." Optik-International Journal for Light and Electron Optics,pp. 1795-1799,2014.

[11] Bhandari, A. K.,"Cuckoo search algorithm based satellite image contrast and brightness enhancement using DWT-SVD." ISA transactions ,pp.1286-1296,2014.

[12] Raju, G., and Madhu S. Nair. "A fast and efficient color image enhancement method based on fuzzylogic and histogram." AEU-International Journal of Electronics and Communications 68,pp. 237243,2014

[13] Li, Jia. "Application of image enhancement method for digital images based on Retinex theory." OptikInternational Journal for Light and Electron Optics ,pp.5986-5988,2014.

[14] Chang, Yung Tseng, "Contrast Enhancement in Palm Bone Image Using Quad-Histogram Equalization." Computer, Consumer and Control (IS3C), 2014 International Symposium on. IEEE, 2014.

[15] N. Senthilkumaran, J. Thimmiaraja, Histogram equalization for image enhancement using mri brain images, in: Computing and Communication Technologies (WCCCT),2014 World Congress on, IEEE, pp. $80-83,2014$

[16] M. S. Hitam, W. N. J. H. W. Yussof, E. A. Awalludin, Z. Bachok, Mixture contrast limited adaptive histogram equalization for underwater image enhancement, in: Computer Applications Technology (ICCAT), 2013 International Conference on, IEEE, pp. 1-5,2014

[17] Selvi, M., and Aloysius George. "FBFET: Fuzzy based fingerprint enhancement technique based on adaptive thresholding." 2013 Fourth International Conference on Computing, Communications and Networking Technologies (ICCCNT). IEEE, 2013.

[18] Suneel, M., K. Kiran Kumar, and P. Uday Bhaskar. "Color Image Enhancement Using Fuzzy Set Theory." Digital Image Processing, pp.10-12,2012.

[19] Z. M. Ramadan, A new method for impulse noise elimination and edge preservation,Electrical and Computer Engineering, Canadian Journal , pp. 2-10,2014. 


\section{Authors}

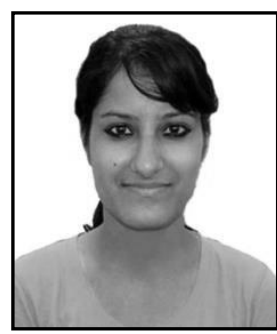

Shruti Puniani, she received the degree of B.Tech in Computer Science and Engineering from Lovely Professional University, Phagwara. Currently, she is pursuing M.Tech in Computer Science and Engineering from DAV University, Jalandhar. Her current research works include Digital Image Processing and its applications.

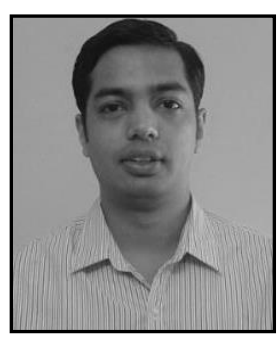

Sankalap Arora, he was born on Nov 3, 1988. He received his Bachelor's degree (B.Tech.) and Master's degree (M.Tech) in Computer Science Engineering from Lovely Professional University, Phagwara, Punjab in year 2011. He is currently pursuing his Doctoral degree (Ph.D.) from Punjab technical University, Kapurthala, Punjab (India). He is currently working as Assistant Professor in DAV University, Jalandhar. His fields of special interest include Evolutionary Algorithms, Artificial Neural Networks, Digital Image processing and Soft Computing for solving engineering problems. He has published several research papers in reputed International Journals and Conferences. 
International Journal of Signal Processing, Image Processing and Pattern Recognition Vol.8, No.8 (2015) 